\title{
Adaptive connectionist systems for engineering applications
}

\author{
Chrisina Jayne
}

Received: 25 October 2013/Accepted: 28 October 2013/Published online: 6 November 2013

(C) Springer-Verlag Berlin Heidelberg 2013

Adaptive Connectionist Systems refer to systems that change with time through interaction with the environment, learning through the utilization of artificial neural networks (ANN). Many engineering applications require the use of real-time decision-making and adaptation to new input. In this context a number of researchers have explored the use of adaptive connectionist systems and their applicability to solve engineering problems.

This special issue includes selected contributions from the 13th Engineering Applications of Neural Network conference held in London, UK in 2012 (EANN 2012).

The EANN conferences promote neural networks and associated techniques and the significant benefits that can be derived from their use. The conferences are not only for reporting advances, but also for showing how neural networks provide practical solutions in a wide range of applications.

The first paper "Neurocomputing techniques to dynamically forecast spatiotemporal air pollution data" by Antonios Papaleonidas and Lazaros Iliadis addresses the application of ANNs to the problem of forecasting dangerous levels of Ozone (O3) concentrations in the city center of Athens and more specifically in the "Athinas" air quality monitoring station. While most existing approaches develop static ANN models optimal only under specific conditions, this work suggests a dynamic flexible and adjustable neural spatiotemporal model. The proposed approach takes in account not only the values of the primitive air pollutants and meteorological measurements,

C. Jayne $(\square)$

Faculty of Engineering and Computing, Coventry University,

Room EC306, Coventry CV1 5FB, UK

e-mail: ab1527@coventry.ac.uk;

Chrisina.Jayne2@coventry.ac.uk but it is also evaluates the influence of the existing $\mathrm{O} 3$ values in monitoring stations close to the one under examination. The results suggest that the optimal ANN model is using data from a small number of measurement's stations and it is capable of estimating $\mathrm{O} 3$ concentrations in real time mod. It also has the capacity of forecasting the same values for future time intervals.

The second paper "Information dynamics based selfadaptive reservoir for delay temporal memory tasks" by Sakyasingha Dasgupta, Florentin Wörgötter and Poramate Manoonpong, investigates the application of recurrent neural networks of the reservoir computing (RC) type for time-series processing tasks and more specifically for delayed response tasks involving the transient memorization of information (temporal memory). The authors combine a generalized intrinsic plasticity rule with a local information dynamics based schema of reservoir neuron leak adaptation. Utilizing this combination they propose a self-adaptive reservoir (RNN) framework that maintains homeostasis through the generic intrinsic plasticity mechanism. The suggested framework has been evaluated on two standard benchmark tasks and the results demonstrate that the adaptation mechanism clearly outperforms static reservoirs. The authors also demonstrate the application of the self-adaptive reservoir (RNN) to the control of autonomous robotic agents through maze navigation experiments. The results show that the proposed network is effective for reconstructing the original trajectories and has the ability to cope with the variable temporal delay memory problem.

The third paper "Exploratory analysis of mobile phone traffic patterns using 1-dimensional Self Organizing Map (SOM), clustering and anomaly detection" by Pekka Kumpulainen, considers the problem of recognizing traffic patterns in continuously evolving mobile networks. This is 
a challenging problem in the telecommunications industry requiring tools capable of intelligent analysis of the network monitoring data. The proposed methods are based on clustering and a one- dimensional self-organizing map. The reason for adopting a one-dimensional map is that this type of map has more compact visualization, which is suitable for data where the variables in the feature vectors are not independent but are derived form meaningful patterns. This work offers analysis of the daily behavior on network element (NE) level by constructing behavioral profiles, which summarize the proportions of distinct daily traffic patterns of each NE. The following procedure for analysis is proposed: preprocessing, day of the week analysis, NE analysis and anomaly detection. The results suggest that this procedure is applicable to the analysis of any other type of traffic, or other variables, that produce repeating periodic patterns.

The fourth paper "Neural Adaptive Control in Application Service Management Environments" by Tomasz D. Sikora and George D. Magoulas, presents a new approach to the application service management (ASM) problem for scalable distributed environments. ASM refers to the monitoring and management of performance and quality of service in complex enterprise systems. An ASM controller needs to react adaptively to changing system conditions in order to optimize a set of Service Level Agreements (SLAs), which operate as objective functions. The proposed approach combines knowledge-bases with neural networks and, unlike previous methods, does not require a model(s) for the enterprise system or the resources. The authors suggest exploiting past data and incremental learning. This incremental learning leads to evolving controller behavior by taking into account consequences of earlier actions in a particular situation, or other similar situations. The authors evaluate the applicability and effectiveness of the proposed neural adaptive controllers using a knowledge base with many gigabytes of system runtime metrics. The empirical results show these controllers are able to adapt to changing runtime conditions based on SLA definitions.

We hope that you will find the papers interesting.

The Guest editor wishes to thank all the authors and reviewers that contributed to this special issue, and to the editors-in-chief and editorial office of the Evolving Systems journal for their support. 